\title{
Study of $\bar{p} p$ annihilation at rest into $\omega \eta \pi^{0}$
}

\author{
Crystal Barrel Collaboration
}

C. Amsler $^{n}$, D.S. Armstrong ${ }^{a}$, I. Augustin ${ }^{g}$ 1, C.A. Baker ${ }^{d}$, B.M. Barnett ${ }^{j}$, C.J. Batty ${ }^{d}$,

K. Beuchert ${ }^{b}$, P. Birien ${ }^{a}$, P. Blüm ${ }^{g}$, R. Bossingham ${ }^{a}$, K. Braune ${ }^{k}$, J. Brose ${ }^{j}$, D.V. Bugg ${ }^{h}$, M. Burchell ${ }^{2}$, T. Case ${ }^{a}$, S.U. Chung ${ }^{3}$, A. Cooper ${ }^{h}$, K.M. Crowe ${ }^{a}$, T. Degener ${ }^{b}$, H.P. Dietz ${ }^{k}$, S. von Dombrowski ${ }^{n}$, M. Doser ${ }^{e}$, W. Dünnweber ${ }^{k}$, D. Engelhardt ${ }^{g}$, M. Englert ${ }^{k}$, M.A. Faessler ${ }^{k}$, C. Felix ${ }^{k}$, R. Hackmann ${ }^{j}$, R.P. Haddock ${ }^{i}$, F.H. Heinsius ${ }^{a}$, N.P. Hessey ${ }^{e}$, P. Hidas ${ }^{c}$, P. Illinger ${ }^{k}$, D. Jamnik ${ }^{k}{ }^{4}$, Z. Jávorfi ${ }^{c}$, H. Kalinowsky ${ }^{j}$, B. Kämmle ${ }^{f}$, T. Kiel ${ }^{g}$, J. Kisiel ${ }^{k}{ }^{5}$, E. Klempt ${ }^{j}{ }^{6}$, M. Kobel ${ }^{e}$, H. Koch ${ }^{b}$, C. Kolo ${ }^{k}$, K. Königsmann ${ }^{k} 8$, M. Kunze $^{b}$, R. Landua ${ }^{e}$, F. Loser ${ }^{f}$, J. Lüdemann ${ }^{b}$, H. Matthäy ${ }^{b}$, M. Merkel ${ }^{j}{ }^{9}$, J.P. Merlo ${ }^{j}$, C.A. Meyer ${ }^{l}$, L. Montanet ${ }^{e}$, A. Noble ${ }^{n}$, F. Ould-Saada ${ }^{n}$, K. Peters ${ }^{b}$, C.N. Pinder ${ }^{d}$, G. Pinter ${ }^{c}$, S. Ravndal ${ }^{b 10}$, J. Salk ${ }^{b}$, A.H. Sanjari ${ }^{h}{ }^{11}$, E. Schäfer ${ }^{j}$, B. Schmid ${ }^{n}{ }^{12}$, P. Schmidt ${ }^{f}$, S. Spanier ${ }^{j}$, C. Straßburger ${ }^{j}{ }^{13}$, U. Strohbusch $^{f}$, M. Suffert ${ }^{m}$, D. Urner ${ }^{n}$, C.Völker ${ }^{k}$, F. Walter ${ }^{f}$, D. Walther ${ }^{b}$, U. Wiedner ${ }^{f}$, N. Winter ${ }^{g}$, J. Zoll ${ }^{e}$, B.S. Zou ${ }^{h}, \breve{C}$. Zupančičc ${ }^{k}$

a University of California, LBL, Berkeley, CA 94720, USA

${ }^{b}$ Universität Bochum, 44780 Bochum, Germany

c Academy of Science, H-1525 Budapest, Hungary

${ }^{d}$ Rutherford Appleton Laboratory, Chilton, Didcot OX11 0QX, UK

e CERN, CH-1211 Genève, Switzerland

${ }^{f}$ Universität Hamburg, 22761 Hamburg, Germany

9 Universität Karlsruhe, 76344 Karlsruhe, Germany

${ }^{h}$ Queen Mary and Westfield College, London E1 4 NS, UK

i University of California, Los Angeles, CA 90024, USA

$j$ Universität Mainz, 55099 Mainz, Germany

${ }^{k}$ Universität München, 85748 München, Germany

$l$ Carnegie Mellon University, Pittsburgh, PA, USA

${ }^{m}$ Centre de Recherches Nucléaires, F-67037 Strasbourg, France

${ }^{n}$ Universität Zürich, CH-8001 Zürich, Switzerland

Received ; revised manuscript received

We have studied $\bar{p}$ p annihilations at rest in liquid hydrogen into the final state $\omega \eta \pi^{0}$ where $\omega$ decays to $\pi^{0} \gamma$. This reaction is dominated by the production of $a_{0}(980)$ and contributions from $a_{2}(1320)$ and $b_{1}^{0}(1235)$. Upper limits for the production of an axial vector $h_{1}^{\prime}(1380)$ meson and vector mesons $\omega(1390), \omega(1600)$, and $\phi(1680)$ decaying to $\omega \eta$ are given. The branching ratio of $\overline{\mathrm{p} p}$ annihilation into $\omega \eta \pi^{0}$ was determined to be $(0.68 \pm 0.01 \pm 0.05) \%$.

\footnotetext{
${ }^{1}$ Now at Universität Siegen, Siegen, Germany

${ }^{2}$ Now at University of Kent, Canterbury, UK

${ }^{3}$ On leave of absence from BNL, Upton, NY, USA

${ }^{4}$ On leave of absence from the University of Ljubljana, Ljubljana, Slovenia
}

\footnotetext{
${ }^{5}$ On leave of absence from the University of Silesia, Katowice, Poland

${ }^{6}$ Now at Universität Bonn, D-55127 Bonn, Germany

${ }^{7}$ Now at Universität Freiburg, D-79104 Freiburg, Germany
} 
The reaction $\overline{\mathrm{p}} \mathrm{p} \rightarrow \omega \eta \pi^{0}$ has not been studied previously due to a lack of experiments having efficient detection for final states with several photons. This process is of interest to search for isoscalar and axial vector mesons decaying to $\omega \eta$, in particular the partner of $h_{1}(1170)$ which is not well established. A candidate decaying into $K^{\star}(895) K$ has been reported at $1380 \mathrm{MeV} / \mathrm{c}^{2}$ [1]. Until now only two states decaying into $\omega \eta$ have been reported. In the reaction $\gamma p \rightarrow \omega \eta p$ from diffractive photoproduction by photons of 25-50 GeV, a signal of unknown spin and parity has been reported at a mass of $1610 \mathrm{MeV} / \mathrm{c}^{2}$ with a width of $230 \mathrm{MeV} / \mathrm{c}^{2}$ [2]. Another signal has been reported at a mass of $1650 \mathrm{MeV} / \mathrm{c}^{2}$ with a width of less than $70 \mathrm{MeV} / \mathrm{c}^{2}$ in the reaction $\pi^{-} \mathrm{p} \rightarrow \omega \eta \mathrm{n}, \omega \eta \rightarrow 5 \gamma$ at a $\pi^{-}$momentum of $38 \mathrm{GeV} / \mathrm{c}$ [3]. In order to further investigate this open issue, we have studied $10,924 \omega \eta \pi^{0}$ events in the reaction $\bar{p} p \rightarrow \omega \eta \pi^{0}$ at rest, where $\omega$ decays into $\pi^{0} \gamma$.

The experiment was performed on beam line $\mathrm{C} 2$ at the Low Energy Antiproton Ring (LEAR) at CERN. Antiprotons with an extracted momentum of $200 \mathrm{MeV} / \mathrm{c}$ are stopped in a $4 \mathrm{~cm}$ long liquid hydrogen target. A matrix of four silicon counters in front of the target defines and monitors the incident $\overline{\mathrm{p}}$ (typically $10^{4} \overline{\mathrm{p}} / \mathrm{s}$ ). The target is surrounded by two cylindrical proportional wire chambers, a jet drift chamber to detect charged particles and a barrel-shaped calorimeter consisting of $1380 \mathrm{CsI}(\mathrm{Tl})$ crystals, 16 radiation lengths deep, with photodiode readout. The CsI calorimeter covers the polar angles between $12^{\circ}$ and $168^{\circ}$ with full coverage in azimuth. The useful acceptance for shower detection is $0.95 \times 4 \pi$ sr. The energy calibration of the calorimeter is obtained by

\footnotetext{
${ }^{8}$ Now at Max-Planck-Institut, Heidelberg, Germany

${ }^{9}$ Now at CERN, Switzerland

${ }^{10}$ This work comprises part of the PhD thesis of S. Ravndal

${ }^{11}$ Now at State University of Stony Brook, NY, USA

${ }^{12}$ Now at University of California, Irvine, USA

${ }^{13}$ Now at Universität Bonn, D-55127 Bonn, Germany
}

Fig. 1. $\pi^{0} \gamma$ mass distribution with two entries/event before (blank) and after (hatched) requiring the energy of the single- $\gamma$ to lie above $180 \mathrm{MeV}$. The inset shows the hatched signal in the region between 700 and $900 \mathrm{MeV} / \mathrm{c}^{2}$ in $1 \mathrm{MeV} / \mathrm{c}^{2}$ bins and the applied fit.

adjusting the calibration factor of each crystal to center the $\pi^{0}$ peak and the $\eta$ peak in the $\gamma \gamma$ invariant mass spectrum at their nominal masses. The energy resolution is $\sigma_{E} / E=2.5 \%$ for $1 \mathrm{GeV}$ photons and the angular resolution is typically $\sigma=20$ mrad in both polar and azimuthal angles. The assembly is located in a solenoidal magnet providing a homogeneous field of $1.5 \mathrm{~T}$ parallel to the incident $\bar{p}$ beam. A more detailed description can be found in Ref. [4].

The data presented here are based on $10.1 \times 10^{6}$ annihilations into neutral events, which were collected by vetoing charged particles online with the proportional wire chambers and the inner layers of the jet drift chamber (0-prong trigger). Events with residual tracks are removed in the offline analysis. Events with seven electromagnetic showers with energy deposits exceeding $20 \mathrm{MeV}$ are then selected, leaving 1,073,596 events. A further selection step requiring the total energy to be in the range of 1700 to $2050 \mathrm{MeV}$ and the total momentum below $100 \mathrm{MeV} / \mathrm{c}$ reduces the number to 
614,452 events. Events with a shower induced in the last crystal rows around the beam entrance and exit pipes are removed to suppress energy leakage. A possible background contribution stems from events with six photons from $\eta \pi^{0} \pi^{0}$, for which one of the showers develops a small neighbouring satellite which is interpreted as an additional shower. These so called 'split-off' events are suppressed by requiring the relative energy deposit of two neighbouring showers, the centroids of which are separated by less than $14^{\circ}$, to be larger than 0.18 . The application of this cut reduces the number of events to 344,596 .

Events are then submitted to kinematic fit to the hypotheses $\pi^{0} \pi^{0} \pi^{0} \gamma(7 \mathrm{C}), \eta \pi^{0} \pi^{0} \gamma(7 \mathrm{C})$, $\omega \pi^{0} \pi^{0}(8 \mathrm{C})$ and $\omega \eta \pi^{0}(8 \mathrm{C})$, where the $\omega$ decays to $\pi^{0} \gamma$ and each $\pi^{0}$ to $\gamma \gamma$. For the channel $\overline{\mathrm{p} p} \rightarrow \eta \pi^{0} \pi^{0} \gamma$ we only accept events with a confidence level $\geq 10 \%$, if the confidence levels of the fits to the $\pi^{0} \pi^{0} \pi^{0} \gamma$ and $\omega \pi^{0} \pi^{0}$ are both below $1 \%$. This yields 23,278 events. For the channel $\bar{p} p \rightarrow \omega \eta \pi^{0}$, one expects the energy of the single-photon from the $\omega$ decay to lie in the range between $196 \mathrm{MeV}$ and $735 \mathrm{MeV}$. The expected energy resolution for a photon with an energy of $196 \mathrm{MeV}$ is $\sigma_{\gamma} \approx 7.3 \mathrm{MeV}$. Figure 1 shows the $\pi^{0} \gamma$ mass distribution before and after requiring a minimum single- $\gamma$ energy of $180 \mathrm{MeV}$ which is a $2 \sigma_{\gamma}$ cut below the lower single-photon energy. The cut removes residual background contribution from the $\eta \pi^{0} \pi^{0}$ final state, which survived the 'split-off' cut applied earlier.

The inset in Figure 1 shows the $\pi^{0} \gamma$ mass distribution in an expanded scale. We applied a fit with a Breit-Wigner function folded with a gaussian (voigtian) to include the experimental resolution, and a second order polynomial for the background. In the fit we fixed the width of the $\omega$ to $8.43 \mathrm{MeV}[5]$ and determined the mass of the $\omega$, the experimental gaussian mass resolution $\sigma_{\pi^{0} \gamma}$ and the number of $\omega$ 's above the combinatorial background. We found $N=(10,662 \pm 127 \pm 141)$ true $\omega \eta \pi^{0}$ events in the peak in the $\omega$ mass region. The $\omega$ mass was determined to be

$m_{\omega}=(781.96 \pm 0.17 \pm 0.80) \mathrm{MeV} / c^{2}$,

which is in good agreement with the world average of $(781.95 \pm 0.14) \mathrm{MeV}$ [5]. For our experimental resolution of the $\pi^{0} \gamma$ mass we found

$\sigma_{\pi^{0} \gamma}=(13.59 \pm 0.18 \pm 1.67) \mathrm{MeV} / c^{2}$

The first uncertainty is statistical and the second stems from the uncertainty in the precise shape of the background for the various data taking periods. The final selection criterion requires the confidence level of the fit to $\omega \eta \pi^{0}(8 \mathrm{C})$ hypothesis to be larger than $10 \%$ for the $\pi^{0} \gamma$ mass combination closest to the nominal $\omega$ mass. This cut leaves 10,924 events accepted as $\omega \eta \pi^{0}$.

To check the quality of the data and to have comparison capabilities we also studied the final state where $\eta$ decays to $\pi^{0} \pi^{0} \pi^{0}$. This leads to eleven detected photons which were selected from a subsample of $4.8 \times 10^{6}$ annihilations with the online 0 -prong trigger. After applying similar selection criteria, removing 'split-off's and rejecting events with hits centered in the crystals adjacent to the beam line, we found 34,826 events. A $9 \mathrm{C}$ kinematic fit was performed to the hypothesis $\pi^{0} \pi^{0} \pi^{0} \pi^{0} \pi^{0} \gamma$. A cut on the confidence level was applied at $10 \%$ and the single- $\gamma$ energy was required to be more than $180 \mathrm{MeV}$. In Figure $2 \mathrm{a}$ we show the $\pi^{0} \gamma$ mass in the region between 700 and $900 \mathrm{MeV} / \mathrm{c}^{2}$. In Figure 2b we show the $\pi^{0} \pi^{0} \pi^{0}$ mass in the region between 520 and $580 \mathrm{MeV} / \mathrm{c}^{2}$. The curves are Voigtian functions with polynomial backgrounds which were fitted to both mass distributions. We found $(3,463 \pm 84 \pm 142) \omega$ events in the $\pi^{0} \gamma$ mass distribution with

$m_{\omega}=(782.08 \pm 0.36 \pm 0.82) \mathrm{MeV} / c^{2}$,

which is again compatible with the value from Ref. [5]. The experimental resolution is

$\sigma_{\pi^{0} \gamma}=(14.09 \pm 0.35 \pm 1.71) \mathrm{MeV} / c^{2}$.

For the $\pi^{0} \pi^{0} \pi^{0}$ mass distribution we observed $(2,232 \pm 108 \pm 7) \eta$ events with

$m_{\eta}=(548.01 \pm 0.07 \pm 1.05) \mathrm{MeV} / c^{2}$,

which is in agreement with the value of $(547.45 \pm$ 0.19) $\mathrm{MeV} / \mathrm{c}^{2}$ from Ref. [5]. This mass determination of the $\eta$ is however not entirely independent since the calibration of the electromagnetic calorimeter centers the peak in the $\gamma \gamma$ invariant 
Fig. 2. a) $\pi^{0} \gamma$-invariant mass distribution ( 5 combinations/event), b) $3 \pi^{0}$-invariant mass distribution (10 combinations/event).

mass spectrum at the nominal mass of the $\eta$. The $11-\gamma$ final state was not used for further analysis due to the lower statistics and higher background.

In order to evaluate the acceptance for $\omega \eta \pi^{0}$ in the seven-photon final state a Monte Carlo study using GEANT3.14 [6] and a full detector simulation was performed. The Monte Carlo events were tracked, reconstructed and submitted to the same cuts as real data. We used $10^{5}$ phase space distributed $\omega \eta \pi^{0}$ events. This led to a detection and reconstruction efficiency $\epsilon=(18.41 \pm 0.15 \pm$ $0.77) \%$. The first error is statistical while the second error includes systematic uncertainties in the simulation (threshold, noise, annihilation outside the target and annihilation at in flight). The acceptance is flat over the $\omega \eta \pi^{0}$ Dalitz plot, except for cells at the periphery. In the partial-wave analysis this effect was taken into account by ignoring the edge cells. In addition we used data samples of $5 \times 10^{4}$ phase-space distributed events of other final states. It turned out the main background contribution was $\eta \pi^{0} \pi^{0} \pi^{0}$ with a missing $\gamma$ from $\pi^{0}$ decay.

In order to correct the data we considered background events from side bins below and above the $\omega$. We selected events with a $\pi^{0} \gamma$ mass $m_{\pi^{0} \gamma}$ from $707 \mathrm{MeV} / c^{2}$ to $737 \mathrm{MeV} / \mathrm{c}^{2}$ and $827 \mathrm{MeV} / \mathrm{c}^{2}$ to $857 \mathrm{MeV} / \mathrm{c}^{2}$. The width of these two regions were equal to the width of the observed $\omega$ signal, see Eq. 2. Let us denote the lower mass of each of these selected regions by $m_{\min }$. We transformed all events in the side bins to have a $\pi^{0} \gamma$ mass of

$m_{\pi^{0} \gamma}^{\prime}=2\left(m_{\pi^{0} \gamma}-m_{\min }\right)+752 \mathrm{MeV} / c^{2}$.

For $\eta \pi^{0} \pi^{0} \gamma$ we then rescaled the momenta of all four particles to match Eq. 6 while conserving total energy and total momentum. It was confirmed in Monte Carlo studies that no phase-space distortion arises from this transformation and subtraction procedure.

Figure 3 shows the $\omega \eta \pi^{0}$ Dalitz plot. The background events have already been subtracted. Figures $4 \mathrm{a}-\mathrm{c}$ show the invariant mass distributions for $\omega \eta, \omega \pi^{0}$ and $\eta \pi^{0}$ after background subtraction. The black histograms show the subtracted background events.

The most prominent peak is visible in the $\eta \pi^{0}$ mass distribution. We identify the peak with $a_{0}(980)$ decaying to $\eta \pi^{0}$. A fit with a Breit-Wigner function folded with a gaussian for the experimental mass resolution and a polynomial to describe the event distribution under the peak was applied. The mass resolution was fixed to the experimental resolution of $\sigma_{\eta \pi^{0}}=11 \mathrm{MeV} / \mathrm{c}^{2}$. We obtain the following mass and width:

$$
\begin{aligned}
m_{a_{0}} & =(984.45 \pm 1.23 \pm 0.34) \mathrm{MeV} / c^{2} \\
\Gamma_{a_{0}} & =(54.12 \pm 0.34 \pm 0.12) \mathrm{MeV} / c^{2}
\end{aligned}
$$

which are compatible with the PDG values [5] of $982.7 \pm 2.0$ and $57 \pm 11 \mathrm{MeV} / \mathrm{c}^{2}$.

The experimental Dalitz plot is analyzed in the helicity formalism in terms of the isobar model in which the $\bar{p}$ p system is assumed to decay to $\omega \eta \pi^{0}$ through sequential two-body decays with amplitudes $A(J L S)$ where $J$ is the spin of the decaying resonance, $L$ the angular momentum of the daughters and $S$ the total spin. A similar analysis with this formalism has been performed in Ref. [7] for the final state $\omega \pi^{0} \pi^{0}$ using the formalism given in [8] and [9], which includes a standard 
Fig. 3. Dalitz plot of the $\omega \eta \pi^{0}$ final state. The population increases from white to black in steps of 20 entries/cell, each being $0.04 \times 0.05 \mathrm{GeV}^{2} / \mathrm{c}^{4}$ wide. The arrows indicate the subdivision of the Dalitz plot into nine different regions.

centrifugal barrier in both production and decay of resonances. Due to parity and $C$-parity conservation the initial state annihilates into $\omega \eta \pi^{0}$ from the ${ }^{3} \mathrm{~S}_{1}\left(J^{P C}=1^{--}\right)$or the ${ }^{1} \mathrm{P}_{1}\left(J^{P C}=1^{+-}\right)$ $\overline{\mathrm{p}} \mathrm{p}$ atomic orbitals following $\overline{\mathrm{p}}$ capture at rest. As will be shown, a good description of the Dalitz plot population was achieved by assuming $\mathbf{S}$-wave dominance in liquid hydrogen [10] and hence neglecting annihilation from the ${ }^{1} \mathrm{P}_{1}$ level. The expected contributions were:

(1) $\overline{\mathrm{p}} \mathrm{p}\left(1^{--}\right) \rightarrow a_{0}(980) \omega$ where $\omega$ is produced with $L=0$ and the $\eta \pi^{0}$ system is in a relative $\mathrm{S}$-wave $(l=0)$. The amplitude for $S=1$ is

$A_{1}=A^{\omega}(111) A^{\overline{\mathrm{p}} \mathrm{p}}(101)$.

$A^{\omega}(111)$ describes the decay $\omega \rightarrow \pi^{0} \gamma$.

(2) $\overline{\mathrm{p}} \mathrm{p}\left(1^{--}\right) \rightarrow a_{2}(1320) \omega$ where $\omega$ is produced with $L=0$ and the $\eta \pi^{0}$ system is in a relative D-wave $(l=2)$. The amplitude is

$A_{2}=\left[A^{\omega}(111) \otimes A^{a_{2}}(220)\right] A^{\overline{\mathrm{p} p}}(101)$

where $\otimes$ denotes a tensor product.
Fig. 4. Invariant mass distributions of a) $\omega \eta$, b) $\omega \pi^{0}$ and c) $\eta \pi^{0}$. The background (black distribution) has already been subtracted. The solid line superimposed is the result of fit $\mathcal{F} 1$. 
(3) $\overline{\mathrm{p}} \mathrm{p}\left(1^{--}\right) \rightarrow b_{1}^{0}(1235) \eta, b_{1}^{0} \rightarrow \omega \pi^{0}$, where $b_{1}^{0}$ is produced with angular momentum $L=0$ or 2 and $\omega$ with angular momentum $l=0$ or 2 . It turns out that the introduction of amplitudes with $L=0$ is sufficient. Therefore, only two amplitudes contribute

$A_{3,4}=A^{\omega}(111) A^{b_{1}^{0}}(1 l 1) A^{\overline{\mathrm{p}}}(101)$.

Contributions from higher angular momenta $(L \geq 2)$ were assumed to be negligible due to phase-space limitation.

The mass and width of $a_{2}$ were fixed at 1318 $\mathrm{MeV} / \mathrm{c}^{2}$ and $110 \mathrm{MeV} / \mathrm{c}^{2}$. The mass and width of $b_{1}^{0}$ were fixed at $1232 \mathrm{MeV} / \mathrm{c}^{2}$ and $155 \mathrm{MeV} / \mathrm{c}^{2}$, see Ref. [5]. For the $\eta \pi^{0} \mathrm{~S}$-wave we chose a BreitWigner description with mass and width according to Eq. 7.

The transition probability $\mathrm{w}$ is given by the $\mathrm{co}^{-}$ herent sum of all amplitudes

$\mathrm{w}=\mathrm{w}_{P S} \times \operatorname{Tr}\left|\sum_{j=1}^{6} a_{j} A_{j}\right|^{2}$

where $w_{P S}$ is the phase-space weight and $a_{j}=\alpha_{j} \exp \left(i \phi_{j}\right)$ are unknown constants. The weight $\mathrm{w}$ can be rewritten in terms of the real constants $\alpha_{j}$ and $\phi_{j}$ to be determined by the fitting procedure:

$$
\begin{aligned}
\mathrm{w}= & \sum_{i} \alpha_{i}^{2} P_{i i} \\
& +2 \sum_{i<j} \alpha_{i} \alpha_{j} \operatorname{Re}\left(P_{i j}\right) \cos \left(\phi_{i}-\phi_{j}\right) \\
& +2 \sum_{i<j} \alpha_{i} \alpha_{j} \operatorname{Im}\left(P_{i j}\right) \sin \left(\phi_{j}-\phi_{i}\right)
\end{aligned}
$$

where

$P_{i j}=\mathrm{w}_{P S} \times \operatorname{Tr}\left(A_{i} A_{j}^{\dagger}\right)$.

One phase $\phi_{j}$ being arbitrary, we chose $\phi_{1}=0$. This led to 6 parameters $\alpha_{j}$ and 5 phases $\phi_{j}$.

The edge bins in the Dalitz plot were excluded from the fit. The angular distribution of the $\gamma$ was used as additional information. In the rest frame of the $\omega$ we define a system with the z-axis in the $\omega$ flight direction. The $\mathrm{y}$-axis is defined by the normal vector to the $\omega \eta \pi^{0}$ decay plane. We calculate the polar angle $\Theta_{\gamma}$ and the azimuthal angle $\Phi_{\gamma}$ of the $\gamma$ in the $\omega$ rest system for each event. The Dalitz plot is divided into nine independent regions, indicated by the arrows in the plot shown in Figure 3. Out of these nine regions we select the six regions $(2,3,5,6,7,8$, see Figure 3$)$ with at least 1000 entries. The decay angles $\Theta_{\gamma}$ and $\Phi_{\gamma}$ were stored in six two dimensional histograms with 100 cells. The background contribution was subtracted by generating angular distributions from the $\omega$ side-bins according to the procedure described above. The Dalitz plot was divided into cells of $0.04 \times 0.05 \mathrm{GeV}^{2} / \mathrm{c}^{4}$ width. Cells with less than 5 entries were not used in the fit. The Dalitz plot and the six angular distribution were fitted simultaneously.

This left for the Dalitz plot 207 cells and 522 cells $\left(\Theta_{\gamma}, \Phi_{\gamma}\right)$ with $N_{T}=9,766 \omega \eta \pi^{0}$ events hence 729 cells in which each event entered only once with the four observables $m_{\omega \eta}, m_{\eta \pi^{0}}, \Theta_{\gamma}$ and $\Phi_{\gamma}$.

The GEANT Monte Carlo used to determine the acceptance of the $\omega \eta \pi^{0}$ final state was not statistically sufficient to permit a meaningful Dalitz plot analysis. Therefore, we generated $3 \times 10^{5}$ $\omega \eta \pi^{0}$ events with a phase-space generator, ignoring detector acceptance and reconstruction efficiency. The $\omega$ width was given by the experimental resolution, Eq. 2. The simulated Dalitz plot population was compared to the corresponding $10^{5}$ GEANT events by using a $\chi^{2}$ test. Both data sets turned out to be consistent, and hence we used the events from the simple phase-space generator for the following amplitude analysis. Monte Carlo Dalitz plots weighted by $P_{i j}$ were generated for each pair of amplitudes $i, j(i \leq j)$. The $P_{i j}$ (Eq. 13) and correspondingly the weight w (Eq. 11), were normalized to the total number of events $N_{T}$ :

$P_{i j} \rightarrow \frac{P_{i j}}{\sqrt{f_{i} f_{j}}}$ with $f_{i}=\sum P_{i i} / N_{T}$

where the sum extends over all $\left(3 \times 10^{5}\right)$ Monte Carlo events. We then build the $\chi^{2}$

$\chi^{2}=\sum \frac{(n-\mathrm{w})^{2}}{n+\sigma_{\mathrm{w}}^{2}}$

where $n$ is the number of entries in the cells of the experimental plots. The sum extends over the contents of the Dalitz plot and the cells of the angular 
Table 1

Fits $\mathcal{F} 2-\mathcal{F} 5$ include additional contributions from Spin 1 mesons.

\begin{tabular}{|c|l|r|r|r|r|r|}
\hline Fit & \multicolumn{1}{|c|}{$\mathcal{F} 1$} & \multicolumn{1}{c|}{$\mathcal{F} 2$} & \multicolumn{1}{c|}{$\mathcal{F} 3$} & \multicolumn{1}{c|}{$\mathcal{F} 4$} & \multicolumn{1}{c|}{$\mathcal{F} 5$} \\
\hline \multicolumn{2}{|c|}{$\chi^{2} /$ DoF } & 1.25 & \multicolumn{1}{c|}{1.20} & \multicolumn{1}{c|}{1.23} & \multicolumn{1}{c|}{1.28} & \multicolumn{1}{c|}{1.19} \\
\hline \multicolumn{2}{|c|}{ Amplitude $A_{i}$} & & $h_{1}^{\prime}(1380)$ & \multicolumn{1}{c}{$\omega(1390)$} & \multicolumn{1}{c}{$\omega(1600)$} & \multicolumn{1}{c|}{$\phi(1680)$} \\
\hline$a_{0} \omega$ & $\alpha_{1}(L=0)$ & $.73 \pm .01$ & $.73 \pm .01$ & $.72 \pm .01$ & $.70 \pm .01$ & $.69 \pm .01$ \\
\hline $\mathrm{a}_{2} \omega$ & $\alpha_{2}(L=0)$ & $.61 \pm .01$ & $.61 \pm .01$ & $.60 \pm .01$ & $.62 \pm .01$ & $.60 \pm .01$ \\
& $\phi_{2}(L=0)$ & $1.98 \pm .08$ & $1.93 \pm .08$ & $2.03 \pm .09$ & $2.08 \pm .08$ & $2.12 \pm .09$ \\
\hline $\mathrm{b}_{1}^{0} \eta$ & $\alpha_{3}(L=0)(l=0)$ & $.31 \pm .02$ & $.33 \pm .02$ & $.29 \pm .02$ & $.30 \pm .02$ & $.30 \pm .02$ \\
& $\alpha_{4}(L=0)(l=2)$ & $.16 \pm .02$ & $.11 \pm .02$ & $.16 \pm .02$ & $.16 \pm .02$ & $.15 \pm .02$ \\
& $\phi_{3}(L=0)(l=0)$ & $4.87 \pm .06$ & $4.71 \pm .06$ & $4.76 \pm .08$ & $4.80 \pm .07$ & $4.67 \pm .07$ \\
& $\phi_{4}(L=0)(l=2)$ & $3.29 \pm .18$ & $3.46 \pm .25$ & $3.21 \pm .23$ & $3.35 \pm .20$ & $3.08 \pm .27$ \\
\hline$A_{i}$ & $\alpha_{A_{1}}$ & & $.07 \pm .02$ & $.08 \pm .02$ & $.06 \pm .02$ & $.13 \pm .01$ \\
& $\alpha_{A_{2}}$ & & $.10 \pm .02$ & & & \\
& $\phi_{A_{1}}$ & & $6.25 \pm .24$ & $.08 \pm .33$ & $.86 \pm .26$ & $1.13 \pm .17$ \\
& $\phi_{A_{2}}$ & & $3.61 \pm .23$ & & & \\
\hline
\end{tabular}

distributions. The $\sigma_{\mathrm{W}}$ are the statistical errors on the Monte Carlo weights, $\sigma_{\mathrm{W}}=\mathrm{w} / \sqrt{n(M C)}$.

We obtained a reduced $\chi^{2} / D o F=1.16$ for 718 degrees of freedom (DoF) (Fit $\mathcal{F} 1$ Table 1$)$. The invariant mass distributions of the data and the fit are compared in Figure 4 where the fit is superimposed as a solid line. The projections of the angular distributions $\left(\Theta_{\gamma}, \Phi_{\gamma}\right)$ are shown in Figure . The shoulder in the $\eta \pi^{0}$ invariant mass spectrum above $1 \mathrm{GeV}^{2} / c^{4}$ (Figure 4c) is due to the tail of the $\mathrm{a}_{2}$. The lower population in the $a_{0}$ band in the Dalitz plot (Figure 3) below $2.1 \mathrm{GeV}^{2} / c^{4}$ in the $\omega \eta$ mass is due to a destructive interference between the $a_{0}$ and the $b_{1}^{0}$ amplitude. The $b_{1}^{0}$ amplitude is also responsible for the weak diagonal structure below the $a_{0}$ band. The destructive interference also explains why $b_{1}^{0}(1235)$ is not directly visible in the mass projection (Figure $4 \mathrm{~b}$ ). The $\mathrm{b}_{1}^{0} \mathrm{am}-$ plitude describes the asymmetry in the $a_{0}$ region rather well. The $D / S$ ratio of $l=2$ to $l=0$ amplitudes for $b_{1}^{0}$ decay can be obtained from the ratio

$D / S=\frac{1}{\sqrt{5}} \frac{\alpha(l=2)}{\alpha(l=0)}$

By using the values of fit $\mathcal{F} 1$ (Table 1 ) we obtained $D / S=0.23 \pm 0.03$. The world average from $\mathrm{b}_{1}$ production in $\pi \mathrm{p}$ and $\gamma \mathrm{p}$ interactions is $0.26 \pm 0.04$ [5].

Additional amplitudes, $\mathcal{A}_{i}$, were introduced in fits $\mathcal{F} 2-\mathcal{F} 5$. Fit $\mathcal{F} 2$ assumed an additional $1^{+-}$ resonance $h_{1}^{\prime}$ decaying to $\omega \eta$ with a width of 80
Table 2

Branching ratios obtained with the results of fit $\mathcal{F} 1$, ignoring interference effects. The first error is statistical and the second is due to systematics. For the $\omega$, all decay modes were taken into account in the calculation of the branching ratios. This is not the case for the decays of the $a_{0}, a_{2}$ and $\mathrm{b}_{1}^{0}$.

\begin{tabular}{lll}
\hline $\mathrm{BR}\left(\overline{\mathrm{p}} \mathrm{p} \rightarrow a_{0} \omega, a_{0} \rightarrow \eta \pi^{0}\right)=$ & $(3.54 \pm .11 \pm .26)$ & $\times 10^{-3}$ \\
$\mathrm{BR}\left(\overline{\mathrm{p}} \mathrm{p} \rightarrow \mathrm{a}_{2} \omega, \mathrm{a}_{2} \rightarrow \eta \pi^{0}\right)=$ & $(2.46 \pm .10 \pm .19)$ & $\times 10^{-3}$ \\
$\mathrm{BR}\left(\overline{\mathrm{p}} \mathrm{p} \rightarrow \mathrm{b}_{1}^{0} \eta, \mathrm{b}_{1}^{0} \rightarrow \omega \pi^{0}\right)=$ & $(7.82 \pm .85 \pm .57)$ & $\times 10^{-4}$ \\
\hline
\end{tabular}

$\mathrm{MeV} / c^{2}$ [1]. In fits $\mathcal{F} 3-\mathcal{F} 5$, we included the $1^{--}$ resonances $\omega(1390), \omega(1600)$ and $\phi(1680)$, with masses and widths taken from Ref. [5]. The data (Figure 4a) seem to require more intensity in the $\omega \eta$ mass region above $1600 \mathrm{MeV} / \mathrm{c}^{2}$. The intensities $\alpha^{2}$ are however not significant with the possible exception of $\phi(1680)$ (Fit $\mathcal{F} 5$ ) the introduction of which improves the $\chi^{2}$. The fit rejects any other spin-parity assignment in this region and prefers $1^{--}$. However, since the excess occurs at the very end of the available phase-space, we prefer to quote upper limits.

Other parametrizations of the $a_{0}$, suggested in Ref. [11], were also tested but asymmetric parametrizations of $a_{0}$ are not favoured by the data.

The data presented here are taken from a sample of 0-prong annihilations. The absolute number of stopped antiprotons and the efficiencies are calculated by reconstructing annihilation events from the channel $\overline{\mathrm{p}} \rightarrow \pi^{0} \pi^{0}$ in the same data set, and 
Table 3

Upper limits ( $95 \%$ confidence level) for the production of Spin 1 mesons decaying to $\omega \eta$.

\begin{tabular}{l}
\hline $\mathrm{BR}\left(\overline{\mathrm{p} p} \rightarrow h_{1}^{\prime}(1380) \pi^{0}, h_{1}^{\prime}(1380) \rightarrow \omega \eta\right)<$ \\
$\mathrm{BR}\left(\overline{\mathrm{p} p} \rightarrow \omega(1390) \pi^{0}, \omega(1390) \rightarrow \omega \eta\right)<0.48 \times 10^{-4}$ \\
$\mathrm{BR}\left(\overline{\mathrm{p} p} \rightarrow \omega(1600) \pi^{0}, \omega(1600) \rightarrow \omega \eta\right)<0.670^{-4}$ \\
$\mathrm{BR}\left(\overline{\mathrm{p} p} \rightarrow \phi(1680) \pi^{0}, \phi(1680) \rightarrow \omega \eta\right)<10^{-4}$ \\
\hline
\end{tabular}

by using the absolute branching ratio for $\pi^{0} \pi^{0}$, $(6.93 \pm 0.43) \times 10^{-4}$, which was determined earlier by this collaboration [12]. With a reconstruction efficiency of $(0.47 \pm 0.02)$ for $\pi^{0} \pi^{0}$ events one finds a total of $N_{\overline{\mathrm{pp}}}=(259.16 \pm 2.38) \times 10^{6}$ annihilations in liquid hydrogen. The branching ratio for $\omega \eta \pi^{0}$ is given by

$$
B R\left(\overline{\mathrm{p}} \mathrm{p} \rightarrow \omega \eta \pi^{0}\right)=\frac{N}{\epsilon B_{\omega} B_{\eta} N_{\overline{\mathrm{p}} \mathrm{p}}},
$$

where $N$ is the number of $\omega \eta \pi^{0}$ events $(10,662 \pm$ $127 \pm 11), \epsilon=(18.41 \pm 0.15 \pm 0.77) \%$ is the reconstruction efficiency, $B_{\omega}$ the branching ratio for $\omega$ decay into $\pi^{0} \gamma(8.5 \pm 0.5) \%$, and $B_{\eta}$ is the branching ratio for $\eta$ decay into $\gamma \gamma(38.9 \pm 0.5) \%$. This leads to the branching ratio

$$
B R\left(\overline{\mathrm{p}} \mathrm{p} \rightarrow \omega \eta \pi^{0}\right)=(0.68 \pm 0.01 \pm 0.05) \%
$$

which is determined for the first time. The branching ratios for annihilation into $a_{0} \omega, \mathrm{a}_{2} \omega$ and $\mathrm{b}_{1}^{0} \omega$ are not uniquely defined due to interferences between the three channels. We define the branching ratio, relative to $\omega \eta \pi^{0}$, as the sum of contributing intensities $\alpha_{i}^{2}$ normalized to the total incoherent intensity $\sum_{i=1}^{6} \alpha_{i}^{2}$, thereby ignoring interference effects. We find, using the amplitudes from fit $\mathcal{F} 1$, the branching ratios listed in Table 2 . The branching ratio for $\mathrm{a}_{2} \omega$, corrected for all decay modes of the $\mathrm{a}_{2}$ assuming a branching ratio for $\mathrm{a}_{2} \rightarrow \eta \pi^{0}$ of $0.145 \pm 0.012$ [5], is $(1.88 \pm 0.08 \pm 0.21) \%$. Upper limits for the production and decay rates of Spin 1 mesons can be given using the amplitudes from Table 1 and the branching ratio for $\omega \eta \pi^{0} \mathrm{Eq}$. (18) (Table 3 ).

Summarizing, we have performed a Dalitz-plot analysis of the annihilation channel $\bar{p} p \rightarrow \omega \eta \pi^{0}$. This channel is dominated by the production of $a_{0}(980)$. This $\eta \pi^{0} \mathrm{~S}$-wave can be described satisfactorily using a Breit-Wigner amplitude. The
Fig. 5. Angular distributions of $\gamma$ in the $\omega$ rest frame. The curves show from top to bottom for the regions $2,3,5,6$ and 7,8 of the Dalitz plot (Figure 3 ) . 
annihilation channel $\overline{\mathrm{p}} \mathrm{p} \rightarrow \mathrm{b}_{1}^{0} \eta$ has been observed for the first time. Additional states decaying into $\omega \pi^{0}$ have are not been observed. Upper limits have been obtained for production of axial vector and vector mesons decaying into $\omega \eta$.

We would like to thank the technical staff of the LEAR machine group and of all the participating institutions for their invaluable contributions to the success of the experiment. We acknowledge financial support from the German Bundesministerium für Forschung und Technologie, the Schweizer Nationalfonds, the British Science and Engineering Research Council and the U.S. Department of Energy (contract No. DE-FG0387ER40323 and DE-AC03-76SF00098). J.K. acknowledges support from the Alexander von Humboldt Foundation and K.K. from the Heisenberg Foundation.

\section{References}

[1] D. Aston et al., Phys. Lett. B201 (1988) 573

[2] M. Atkinson et al., Z Phys. C34 (1987) 303

[3] G. L. Landsberg (GAMS Collaboration), Proc. Hadron 91 Conference, Maryland, World Scientific, ed. by S. Oneda and D.C. Peaslee, (1992) 12

[4] E. Aker et al., 'The Crystal Barrel Collaboration', Nucl. Instrum. Methods A321 (1992) 69

[5] Review of Particle Properties, Phys. Rev. D45, Part II (1992)

[6] R. Brun et al., Ann. Internal Report CERN DD/EE/84-1, CERN (1987)

[7] C. Amsler et al., 'The Crystal Barrel Collaboration', Phys. Lett. B311 (1993) 362

[8] C. Amsler and J.C. Bizot, Comp. Phys. Comm 30 (1983) 21

[9] F.v. Hippel and C. Quigg, Phys. Rev. D5 (1972) 624

[10] G. Reifenröther and E. Klempt, Phys. Lett. B245, (1990) 129

[11] R.N. Cahn and P.V. Landshoff, Nucl. Phys. B266 (1986) 451

[12] C. Amsler et al., 'The Crystal Barrel Collaboration', Phys. Lett. B297 (1992) 214 